\title{
Effect of Hardness and Stress Ratio on Threshold Stress Intensity Factor Ranges for Small Cracks and Long Cracks in Spheroidal Cast Irons*
}

\author{
Junichiro YAMABE $^{* *}$ and Motokazu KOBAYASHI* \\ ${ }^{* *}$ Mitsubishi Fuso Truck and Bus Corporation \\ 10 Ohkura-cho, Nakahara-ku, Kawasaki-shi 211-8522, Japan \\ junichiro.yamabe@mitsubishi-fuso.com
}

\begin{abstract}
Threshold stress intensity factor ranges, $\Delta K_{t h} \mathrm{~s}$, of spheroidal cast iron, which are composed of ferritic, ferrite-pearlitic, or pearlitic structures, have been estimated by $\Delta K$-increasing tests for small cracks and by $\Delta K$-increasing and $\Delta K$-decreasing tests for long cracks at several stress ratios. With respect to the effects of the hardness and stress ratio, $\Delta K_{\text {th }}$ estimated by the $\Delta K$-decreasing tests for long cracks is evidently different from that for small cracks, while $\Delta K_{\text {th }}$ estimated by the $\Delta K$ increasing tests for long cracks is similar to that for small cracks. Moreover, in the case of long cracks, the values of $\Delta K_{t h}$ estimated by the $\Delta K$-decreasing tests were larger than those estimated by the $\Delta K$-increasing tests, irrespective of the type of matrix structures. These tendencies are attributed to the fact that the crack closure stress of the long cracks in the $\Delta K$-decreasing tests is relatively higher than that of the long cracks in the $\Delta K$-increasing tests. On the fracture surface of the long cracks in the $\Delta K$-decreasing tests, oxide layers produced by fretting are observed at sites around the crack tip, although such layers are not observed on the fracture surface in the $\Delta K$-increasing tests.
\end{abstract}

Key words: Fatigue Limit, Vickers Hardness, Stress Ratio, Threshold Stress Intensity Factor Range, Effective Stress Intensity Factor Range, Crack Closure, Small Crack, Long Crack, Spheroidal Cast Iron

\section{Introduction}

The fatigue strength of spheroidal cast irons is generally lower than that of steels, whose hardness is nearly equal to that of the spheroidal cast irons. This is because the structure of spheroidal cast irons contains graphite and various types of defects. In particular, it is difficult to predict fatigue limit of spheroidal cast irons with cast surfaces due to the complex effects of surface roughness, transitional layers, defects, and residual stress. To solve this problem, we have conducted plane-bending and tension-compression fatigue tests ${ }^{(1)(2)}$ by using specimens with cast surfaces whose residual stress is relieved by annealing in a vacuum. We have also quantitatively evaluated the effects of the above factors excluding the residual stress on the fatigue limit by using the $\sqrt{\text { area }}$ parameter model proposed by Murakami et al ${ }^{(3)-(5)}$. However, with respect to the actual parts, the effect of the residual stress on the fatigue strength must be considered in addition to these factors. Based on the $\sqrt{\text { area }}$ parameter model, the threshold stress intensity factor range, $\Delta K_{t h}$, and fatigue limit, $\sigma_{w}$, at a stress ratio of $R=-1$ are expressed using the defect size, $\sqrt{\text { area }}$, as follows:

$$
\begin{aligned}
【 \sqrt{\text { area }} & <1000 \mu \mathrm{m} 】 \\
\Delta K_{\text {th }} & \cong 3.3 \times 10^{-3}(H V+120)(\sqrt{\text { area }})^{1 / 3}
\end{aligned}
$$


$\sigma_{w}=C \times(H V+120) /(\sqrt{\text { area }})^{1 / 6}$

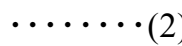

$C=\left\{\begin{array}{l}1.43 \text { (surface defects) } \\ 1.56 \text { (subsurface defects) }\end{array}\right.$

【 $\sqrt{\text { area }} \geq 1000 \mu \mathrm{m} 】$

$\Delta K_{t h}=\Delta K_{t h, \infty}=$ material constant

$\sigma_{w}=\frac{\Delta K_{t h, \infty}}{2 F \times \sqrt{\pi \sqrt{\text { area }} \times 10^{-6}}}$

$F=\left\{\begin{array}{l}0.65 \text { (surface defects) } \\ 0.50 \text { (subsurface defects) }\end{array}\right.$

where, $\Delta K_{t h}$, is the threshold stress intensity factor $[\mathrm{MPa} \sqrt{\mathrm{m}}] ; H V$, the Vickers hardness $\left[\mathrm{kgf} / \mathrm{mm}^{2}\right]$; area, the area projected on the principal plane $\left[\mu \mathrm{m}^{2}\right] ; \sigma_{w}$, the fatigue limit $[\mathrm{MPa}] ; \Delta K_{t h, \infty}$, the threshold stress intensity factor for long cracks [ $\left.\mathrm{MPa} \sqrt{\mathrm{m}}\right]$. Since the fatigue cracks of spheroidal cast irons with cast surfaces sometimes begin from a cast defect with $\sqrt{\text { area }}>1000 \mu \mathrm{m}$, a quantitative evaluation of their fatigue limit must be performed using the accurate value of $\Delta K_{t h, \infty}$. In addition, it is important to clarify the applicable range of Eq. (1) with respect to $\sqrt{\text { area }}$. Although the value of $\Delta K_{t h, \infty}$ is generally estimated by $\Delta K$-decreasing tests, several studies have reported that this value is different from that obtained by the $\Delta K$-increasing tests ${ }^{(6)(7)}$. Therefore, the value of $\Delta K_{t h, \infty}$ should be estimated by $\Delta K$-increasing tests. Thus far, however, the value of $\Delta K_{t h, \infty}$ estimated by $\Delta K$-decreasing tests has hardly been compared with that estimated by $\Delta K$-increasing tests.

Furthermore, we focus on the effect of the stress ratio on the fatigue strengths of specimens with small cracks $(\sqrt{\text { area }} \leq 1000 \mu \mathrm{m})$ and long cracks $(\sqrt{\text { area }}>1000 \mu \mathrm{m})$. Since the actual parts have compressive residual stresses at sites near the cast surfaces produced by shot blasting, we must clarify not only the usefulness and the applicable defect sizes of Eq. (1) but also the effect of the stress ratio on the fatigue strength. Based on the $\sqrt{\text { area }}$ parameter model, the fatigue limit of specimens with small cracks considering the effect of the mean stress is expressed as follows:

$$
\begin{aligned}
& \sigma_{w}=C \times(H V+120) /(\sqrt{\text { area }})^{1 / 6} \cdot[(1-R) / 2]^{\alpha} \\
& \alpha=0.226+H V \times 10^{-4}
\end{aligned}
$$

The error in Eq. (7) is reported to be within $\pm 15 \%$ for materials with $H V=100-740$ ( $\alpha=0.236-0.3$ ). However, Kondo et al. ${ }^{(7)}$ have reported that the values of $\alpha$ for S25C and SCM435 tend to be larger (S25C: $\alpha=0.3$, SCM435: $\alpha=0.8$ ) than those calculated using Eq. (7), although experimental data are insufficient. Therefore, it is also necessary to verify the accuracy of Eq. (7). On the other hand, several evaluation equations using $\Delta K_{t h, R=0, \infty}$, i.e., $\Delta K_{t h, \infty}$ with $R=0$, are proposed ${ }^{(8)-(11)}$ for specimens with long cracks. The value of $\alpha$ for long cracks estimated by $\Delta K$-decreasing tests is in the range of $0.7-1.0^{(8)(9)(12)(13)}$ and these values are considerably larger than those for small cracks.

From this viewpoint, the purpose of this study is to verify the effects of the hardness and stress ratio on the values of $\Delta K_{t h}$ for several types of spheroidal cast irons with small or long cracks and clarify the difference in the values obtained by $\Delta K$-increasing and $\Delta K$ -decreasing tests. To achieve this purpose, we conduct tension-compression fatigue tests using specimens with an artificial defect $(\sqrt{\text { area }} \approx 300-4000 \mu \mathrm{m})$ or a pre-crack and crack growth tests using specimens with a center crack. Moreover, the crack closure behavior of specimens subjected to the $\Delta K$-increasing and $\Delta K$-decreasing tests is observed by applying the unloading elastic compliance method.

2. Materials, specimens, and experimental method

\subsection{Materials}


Table 1 shows the conditions for conducting the fatigue tests on the cast materials. Three types of spheroidal cast irons are employed whose matrices are composed of ferrite (F), ferrite-pearlite (FP), and pearlite $(\mathrm{P})$ structures. A flat plate $\left(40 \times 200 \times 10.5^{\mathrm{t}} \mathrm{mm}\right)$ and Y-block specimens were cast as a row material. Table 2 shows the mechanical properties of these materials. In the case of the $\mathrm{F}$ and $\mathrm{P}$ materials, the flat plate specimens can be considered to be the same as the Y-block specimens because their mechanical properties and the amount of graphite in their microstructures are nearly the same. In the case of the FP material, although the proof stress, tensile strength, and hardness of the flat plate specimens are approximately $10 \%$ higher than those of the Y-block specimens, the amount of graphite in the flat plate specimens is equivalent to that in the Y-block specimens. Therefore, for convenience, these specimens considered to be the same.

\subsection{Specimens}

Figure 1 shows the specimens for the crack growth tests and tension-compression fatigue tests. All the specimens have a smooth surface finish achieved by buffing after emery polishing. The artificial defects shown in Figs.1 (a) and (b), and (c) have been introduced by machining, while those shown in Fig. 1 (c) have been introduced by electrical discharge machining. Then, residual stresses produced by machining are relieved by annealing in a vacuum at $550^{\circ} \mathrm{C}$ for $2 \mathrm{~h}$.

\subsection{Experimental method}

\subsubsection{Crack growth test}

The crack growth tests were conducted with a stress ratio of $R=-1$ and a frequency of 15-30 Hz under loading control, based on ASTM E647-95a ${ }^{(14)}$. In order to eliminate the bending stress generated in the crack growth tests, strain gages were attached to both sides of the specimens, and the alignment was carefully adjusted. The values of $\Delta K_{t h}$ were measured by decreasing $\Delta K$ gradually, after the introduction of a pre-crack at a length of approximately $2 \mathrm{~mm}$ by the application of stress that did not exceed $K_{\max }$ at the beginning of the tests. The crack growth tests with $R=-1$ began at the same level of $K_{\max }$ under the consideration that the values of $\Delta K_{t h}$ depend on those of $K_{\max }$ at the beginning of the tests (15). The crack length was measured using a movable microscope. By employing the secant method, the crack growth rate was estimated from the number of cycles that are required for crack propagation over a length of $500 \mu \mathrm{m}$. The $\Delta K$-decreasing tests were conducted under the conditions that satisfy Eq. (8), and we inferred that crack propagation stopped when $d a / d N<1 \times 10^{-10}[\mathrm{~m} /$ cycle $]$.

Table 1 Condition of fatigue tests

\begin{tabular}{c|c|c||c|c}
\hline \multicolumn{2}{c|}{ Cast material } & \multirow{2}{*}{ Crack growth test ${ }^{* 1}$} & \multicolumn{2}{c}{ Tension-compression fatigue test } \\
\cline { 4 - 5 } & & Artificial defects & Single edge crack ${ }^{* 1}$ \\
\hline \multirow{2}{*}{ FP } & Flat plate & No $^{* 2}$ & $R=-4,-2,-1^{* 3}, 0,0.3$ & No \\
\cline { 2 - 4 } & Y block & $R=-1.5,-1,0.05$ & No & $R=-1.5,-1$ \\
\hline \multirow{2}{*}{ F } & Flat plate & No & $R=-1$ & \multirow{2}{*}{ No } \\
\cline { 2 - 4 } & Y block & $R=-1$ & No & \multirow{2}{*}{ No } \\
\hline \multirow{2}{*}{ P } & Flat plate & No & $R=-1^{* 3}$ & No \\
\cline { 2 - 4 } & Y block & $R=-1$ & Non
\end{tabular}

*1: Crack closure was observed by the unloading elastic compliance method.

*2: The "No" means that a test was not conducted.

*3: These data were taken from an early study. ${ }^{(1)}$

Table 2 Mechanical properties

\begin{tabular}{c|c|c|c|c|c}
\hline \multicolumn{2}{c|}{ Cast material } & $\begin{array}{c}0.2 \% \text { P.S. } \\
{[\mathrm{MPa}]}\end{array}$ & $\begin{array}{c}\text { T.S. } \\
{[\mathrm{MPa}]}\end{array}$ & $\begin{array}{c}\text { Elong. } \\
{[\%]}\end{array}$ & $\begin{array}{c}H V 0.1 \\
{\left[\mathrm{kgf} / \mathrm{mm}^{2}\right]}\end{array}$ \\
\hline \multirow{2}{*}{ FP } & Flat plate & 363 & 555 & 8 & 227 \\
\cline { 2 - 6 } & Y block & 337 & 512 & 14 & 207 \\
\hline \multirow{2}{*}{ F } & Flat plate & 292 & 430 & 23 & 166 \\
\cline { 2 - 6 } & Y block & 290 & 424 & 26 & 160 \\
\hline \multirow{2}{*}{ P } & Flat plate & 462 & 743 & 6 & 286 \\
\cline { 2 - 6 } & Y block & 469 & 761 & 5 & 292 \\
\hline
\end{tabular}




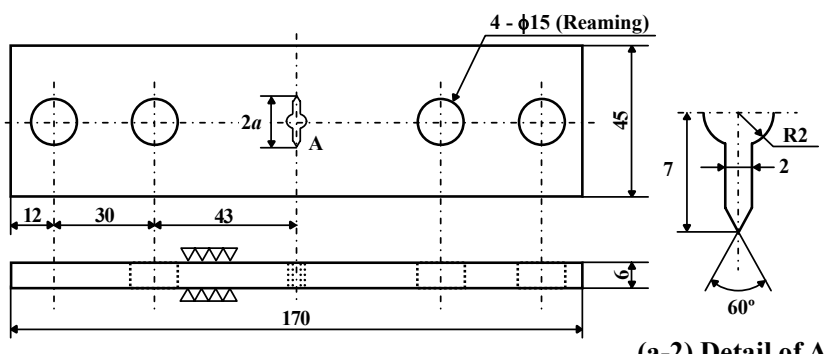

(a-1) Shape and dimension of specimen

(a-2) Detail of $A$

(a) Specimen with a center crack for crack growth tests

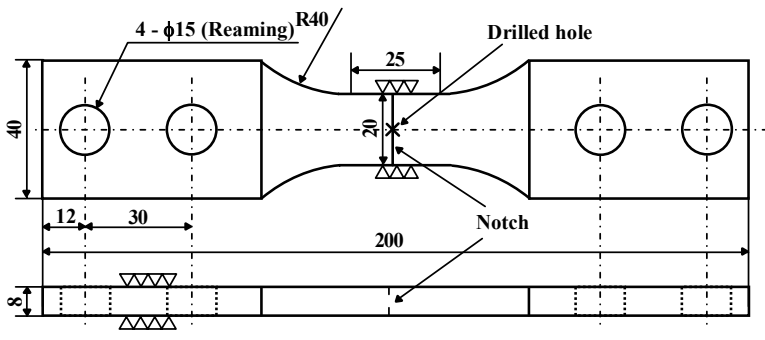

(b-1) Shape and dimension of specimen
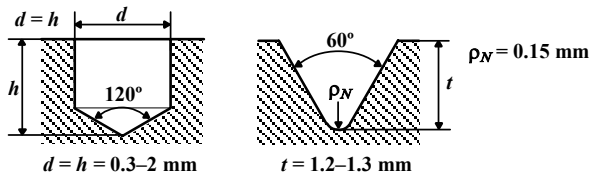

(b-2) Drilled hole

(b-3) Notch

(b) Specimen with an artificial defect for tension-compression fatigue tests
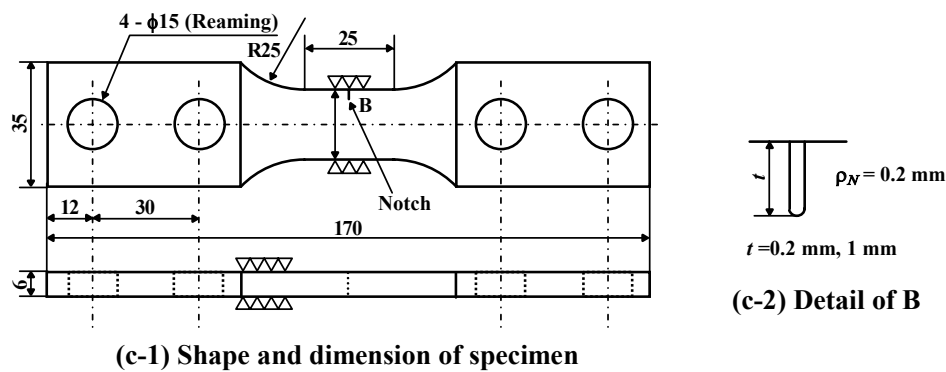

(c-2) Detail of B

(c) Specimen with a single edge crack for tension-compression fatigue tests

Fig.1 Shape and dimensions in $\mathrm{mm}$ of specimens

$$
\frac{1}{\Delta K} \frac{d(\Delta K)}{d a}=-0.08 \pm 0.01\left[\mathrm{~mm}^{-1}\right]
$$

After measuring the values of $\Delta K_{t h}$, crack growth tests accompanied by the application of a constant stress were conducted until the crack length satisfied the condition of small scale yielding expressed in Eq. (9), and the data for middle crack growth rates were measured:

$$
W-2 a \geq 1.25 \frac{P_{\max }}{B \sigma_{y}}
$$

where $P_{\max }$ is the maximum load [N] and $\sigma_{y}$ is the yield stress [MPa]. The crack closure load, $P_{c l}$, was measured by the unloading elastic compliance method in which strain gages were attached at lengths of $3 \mathrm{~mm}$ and $7 \mathrm{~mm}$ from the tip of the notch and these strain gauges are different from those used for adjusting the alignment. Figure 2 shows an example of a compliance curve. A variation point of this curve was considered as the value of $P_{c l}$. The strain gages for obtaining the compliance curve were used under minimum loading to prevent them from slackening. 


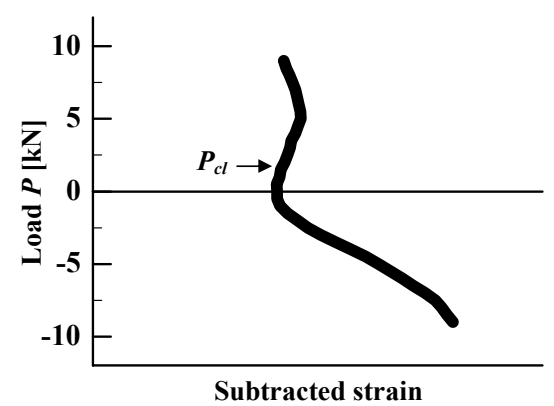

Fig.2 Measurement of crack closure load $P_{c l}$ by the unloading elastic compliance method (Notch depth $t=1 \mathrm{~mm}, \operatorname{Load} P= \pm 9 \mathrm{kN}$ )

\subsubsection{Tension-compression fatigue test}

The tension-compression fatigue tests were conducted on the specimen at the stress ratio of $R=-1$ and a frequency of $10-35 \mathrm{~Hz}$ under loading control, as shown in Figs. 1 (b) and (c). Strain gages were applied on both sides of the specimens and the alignment was carefully adjusted. The fatigue limit of the specimen shown in Fig.1 (b) was defined as the maximum value of the stress amplitude applied to the specimens at which a non-propagating crack was observed at $N=1 \times 10^{7}$. The non-propagating crack in the specimen with a drilled hole was observed by using an optical microscope. On the other hand, the specimen with a notch was compulsorily fractured at room or liquid nitrogen temperature and the existence of a non-propagating crack was determined by observing its fracture surface using a scanning electron microscope. The fatigue limit of the specimens in which the nonpropagating crack cannot be observed was defined as the average of the minimum stress amplitude for broken specimens and the maximum stress amplitude for unbroken specimens.

The tension-compression fatigue tests were conducted on the specimen shown in Fig. 1 (c) after the introduction of a pre-crack at the tip of the notch. To prevent any increase in the crack closure load, the pre-crack was introduced under a high compressive mean stress. The length of the pre-crack for the $200 \mu \mathrm{m}$ notch was $70 \mu \mathrm{m}$, while that for the $1 \mathrm{~mm}$ notch was $150 \mu \mathrm{m}$. Moreover, the crack growth behavior during the fatigue tests was observed using replicas. The values of $P_{c l}$ were measured by applying the unloading elastic compliance method.

\section{Calculation of stress intensity factor ranges}

The value of $\Delta K[\mathrm{MPa} \sqrt{\mathrm{m}}]$ for each specimen was calculated as follows:

【Specimen with a center crack】 ${ }^{(14)}$

$$
\Delta K=\frac{\Delta P}{B} \sqrt{\frac{\pi \beta}{2 W} \sec \frac{\pi \beta}{2}}, \quad \beta=\frac{2 a}{W}
$$

【Specimen with a drilled hole】 ${ }^{(5)}$

$$
\Delta K=0.65 \times 2 \sigma_{a} \sqrt{\pi \sqrt{\text { area }} \times 10^{-6}}
$$

【Specimen with notches on both sides】 ${ }^{(17)}$

$$
\left\{\begin{array}{l}
\Delta K=F(\chi) \times 2 \sigma_{a} \sqrt{\pi t}, \quad \chi=\frac{2 t}{W} \\
F(\chi)=\left\{1+0.112 \cos ^{2}\left(\frac{\pi \chi}{2}\right)\right\} \sqrt{\frac{2}{\pi \chi} \tan \frac{\pi \chi}{2}}
\end{array}\right.
$$

【Specimen with a notch】 ${ }^{(17)}$

$$
\left\{\begin{array}{l}
\Delta K=F(\eta) \times 2 \sigma_{a} \sqrt{\pi t}, \quad \eta=\frac{t}{W} \\
F(\eta)=1.12-0.231 \eta+10.55 \eta^{2}-21.72 \eta^{3}+30.39 \eta^{4}
\end{array}\right.
$$

where $\Delta P$ is the load range $[\mathrm{N}] ; W$, the width of specimen $[\mathrm{m}] ; B$, the thickness of the 
specimen $[\mathrm{m}] ; a$, the crack length $[\mathrm{m}] ; \sigma_{a}$, the stress amplitude $[\mathrm{MPa}] ; \sqrt{\text { area }}$, the defect size $[\mu \mathrm{m}]$; and $t$, the crack depth [m]. The value of $a$ (or $t$ ) is equal to the crack length (or depth) that includes the notch length (or depth).

\section{Results and discussion}

\subsection{Crack growth test}

Figure 3 (a) shows the relationship between the crack growth rate, $d a / d N$, and $\Delta K$. Although the values of $d a / d N$ are similar at high and middle levels of $\Delta K(d a / d N \geq$ $1 \times 10^{-8}[\mathrm{~m} /$ cycle] $)$ for each material with $R=-1$, those at low levels of $\Delta K(\mathrm{da} / \mathrm{dN}<$ $1 \times 10^{-8}[\mathrm{~m} /$ cycle] $)$ are evidently different, i.e., the crack growth rate of the $\mathrm{P}$ material is the fastest and that of the F material is the slowest. Ogawa ${ }^{(18)}$ and Sugiyama ${ }^{(19)}$ have compared the crack growth behavior at low levels of $\Delta K$ by using several types of spheroidal cast irons. The tendency of our data is similar to that of Ogawa's result. Based on the data obtained in our study on the effects of the stress ratio on the crack growth behavior. we observe that the lower the stress ratio, the slower is the crack growth. The threshold stress intensity factor range, $\Delta K_{t h}$, is larger as the stress ratio is lower.

Figure 3 (b) shows the relationship between $d a / d N$ and the effective stress intensity factor range, $\Delta K_{\text {eff. }}$ The relationship between $d a / d N$ and $\Delta K_{\text {eff }}$ for all the materials is successfully shown using a single curve. Therefore, the difference in $d a / d N$ obtained from several structures and stress ratios can be explained by considering the effects of crack closure.

Figure 4 shows the relationship between $\Delta K_{t h}, \Delta K_{\text {eff,th }}$, and $H V$. For the specimens with a long crack, the values of $\Delta K_{t h}$ obtained by $\Delta K$-decreasing tests were reduced because the material was soft. In contrast, the values of $\Delta K_{\text {eff,th }}$ are almost equal for all the specimens, despite the difference in the hardness.

Figure 5 shows the relationship between $\Delta K_{t h}, \Delta K_{\text {eff.th }}$, and $R$ for the FP material shown in Fig. 3. In this figure, the value of $(1-R) / 2$ are plotted on the horizontal axis. For the specimens with a long crack, the values of $\Delta K_{t h}$ obtained by the $\Delta K$-decreasing tests increase with a decrease in the stress ratio, i. e., the values of $(1-R) / 2$ increase. In contrast, the values of $\Delta K_{\text {eff,th }}$ hardly depend on the stress ratio. Furthermore, the values of $\Delta K_{t h}$ are obtained using the following equation:

$$
\Delta K_{t h} \propto\left(\frac{1-R}{2}\right)^{\alpha}
$$

As for the FP material, the value of $\alpha$ is 0.77 for long cracks in the $\Delta K$-decreasing tests, and it is larger than the value for small cracks, which is 0.247 ( $H V=207$ ) calculated using Eq. (7).

\subsection{Tension-compression fatigue test}

Figure 6 shows the relationship between $\Delta K_{\text {th }}$ and $\sqrt{\text { area }}$ obtained from the tensioncompression fatigue tests conducted by gradually increasing $\Delta K^{(1)}$. The values of $\Delta K_{t h}$ for

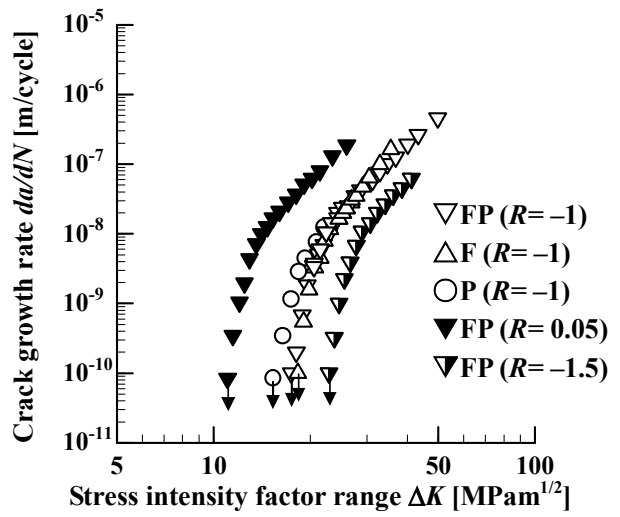

(a) Relationship between $d a / d N$ and $\Delta K$

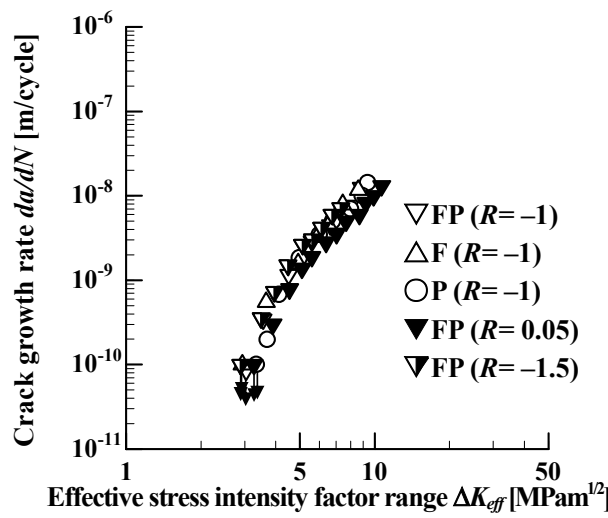

(b) Relationship between $d a / d N$ and $\Delta K_{e f f}$

Fig.3 Relationship between $d a / d N$ and $\Delta K$ or $\Delta K_{\text {eff }}$ 
small cracks were accurately estimated using Eq. (1), which is applicable to specimens with $\sqrt{\text { area }}>1000 \mu \mathrm{m}$ for all the materials. The range of $\sqrt{\text { area }}$ in which Eq. (1) can be applied increases because the material is soft.

Figure 7 shows the relationship between the fatigue limit, $\sigma_{w}$, and the mean stress, $\sigma_{\mathrm{m}}$, for the FP material with a small or long crack. Although the amount of data is insufficient, on the basis of the stress ratio obtained from this study, it is inferred that the effect of the mean stress on the fatigue limit is successfully determined using the modified Goodman diagram.

Figure 8 shows the relationship between $\Delta K_{t h}$ and $H V$ for specimens with small cracks ( $\sqrt{\text { area }}=555 \mu \mathrm{m})$ and long cracks $(\sqrt{\text { area }} \approx 4000 \mu \mathrm{m})$ obtained by the tension-compression fatigue tests. The values of $\Delta K_{\text {th }}$ for small cracks are directly related to those of $H V$ shown in Eq. (1). The values of $\Delta K_{t h}$ for long cracks are also directly related to $H V$, and their tendency differs from that of values for long cracks obtained by the $\Delta K$-decreasing tests, as shown in Fig. 4. For the specimen with a long crack, the difference between the $\Delta K_{t h}$ values obtained by the $\Delta K$-increasing and $\Delta K$-decreasing tests is the highest for the $\mathrm{F}$ material, which is the softest material used in this study. Moreover, the value of $\Delta K_{t h, \infty}$ of the $\mathrm{F}$ material obtained by the $\Delta K$-decreasing tests is 1.4 times larger than that obtained by the $\Delta K$-increasing tests.

Figure 9 shows the relationship between $\Delta K_{\text {th }}$ and $R$ for specimens having small cracks $(\sqrt{\text { area }}=555 \mu \mathrm{m})$ and long cracks $(\sqrt{\text { area }}=1850 \mu \mathrm{m})$ with regard to the FP material in the tension-compression fatigue tests. In this figure, the values of $(1-R) / 2$ are plotted on the horizontal axis, as in Fig. 5. By using Eq. (14), the values of $\alpha$, for small and long cracks are estimated as 0.32 and 0.38 , respectively. These values are nearly equal and higher than the value calculated using Eq. (7), which is 0.247 . The difference between the values of $\Delta K_{t h}$ estimated from the experimental values of a and the calculated of $\alpha(\alpha=0.247)$ is at most $7 \%$ for small cracks and $13 \%$ for long cracks, within the range of the stress ratios tested

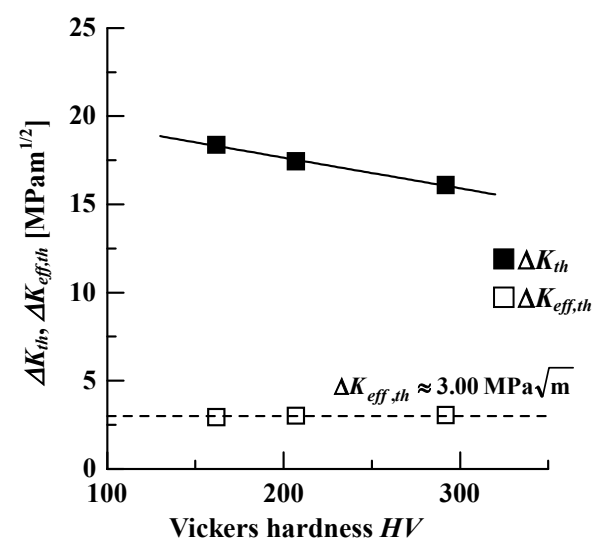

Fig.4 Relationship between $\Delta K_{\text {th }}, \Delta K_{\text {eff,th }}$ and $H V$ for crack growth tests at $R=-1$

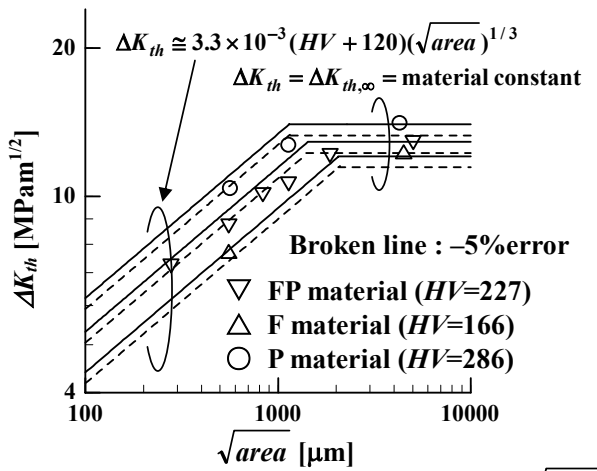

Fig.6 Relationship between $\Delta K_{\text {th }}$ and $\sqrt{\text { area }}$ for tension-compression fatigue tests at $R=-1$

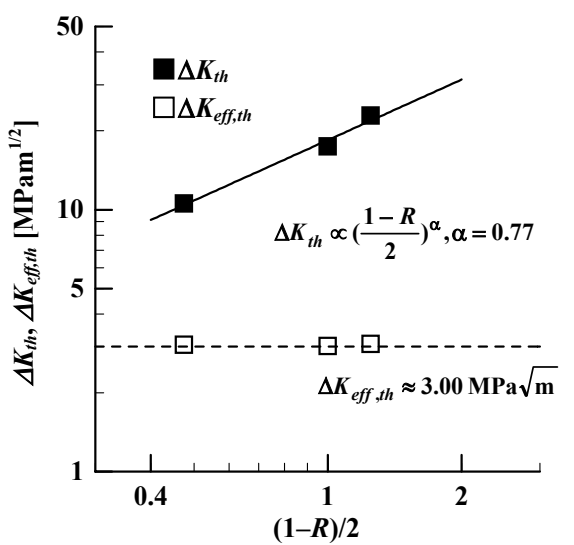

Fig.5 Relationship between $\Delta K_{\text {th }}, \Delta K_{\text {eff th }}$ and stress ratio $R$ for crack growth tests

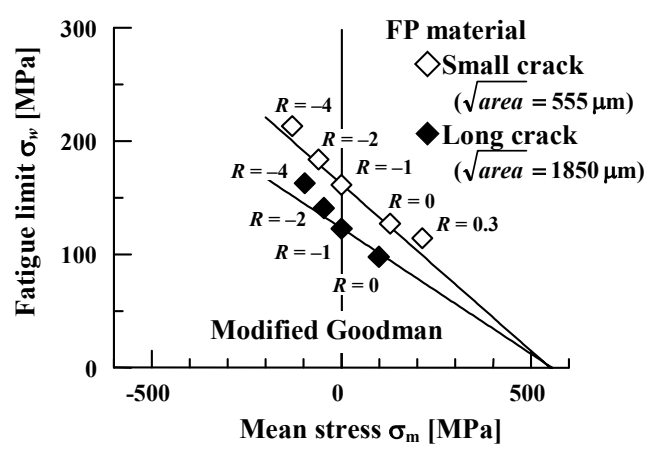

Fig.7 Fatigue limit diagram of FP material containing an artificial defect 
in this study. In other words, $R=-4-0.3$ for small cracks and $R=-4-0$ for long cracks. Therefore, it is considered that the effect of the stress ratio on $\Delta K_{t h}$ can be evaluated using Eq. (7) with sufficient accuracy for practical use within the range of the stress ratios tested in this study. In addition, Eq. (7) can be applied to both small and long cracks. On the other hand, the value of $\alpha$ obtained by the $\Delta K$-decreasing tests (broken line in Fig. 9) is considerably larger than that obtained by the $\Delta K$-increasing tests. It is evident that the tendency of $\Delta K_{t h, \infty}$ obtained by the $\Delta K$-decreasing tests is different from that of $\Delta K_{t h, \infty}$ obtained by the $\Delta K$ increasing tests.

\subsection{Observations of crack closure in tension-compression fatigue tests}

Figure 10 shows the crack closure behavior of the FP material with a long crack (notch length: $t=1 \mathrm{~mm}$ ) obtained by the unloading elastic compliance method. The specimen shown in Fig. 1(c) was tested under tension-compression loading. The fatigue test is conducted at $R=-1$ by applying a stress corresponding to the fatigue limit. In Fig.10, $t^{\prime}$ represents the crack length obtained byadding the notch length $t$ to the crack length $c$ emanating from the tip of the notch. The crack closure load, $P_{c l}$, at $N=1$ is relatively low, because a pre-crack was introduced under a compressive mean stress. However, the value of $P_{c l}$ increases with the number of cycles. Simultaneously, the crack propagates to some extent and then ceases to propagate further. Therefore, the value of $P_{c l}$ at $N=1 \times 10^{7}$ is increases considerably, and the crack closure is generated in a wide range of cyclic loading. Table 3 shows the values of $\Delta K_{t h}, K_{c l, t h}$, and $\Delta K_{\text {eff }, t h}$ for the specimens with small and long cracks at $N=1 \times 10^{7}$ obtained from the fatigue tests. The values of the specimens with long cracks at $R=-1$ and -1.5 are higher than those of the speciemen with small cracks. However, since the values of $\Delta K_{\text {eff,th }}$ for these specimens are nearly equal, we believe that the values of $\Delta K_{\text {eff } f, t h}$ are not influenced by the crack length and stress ratio. The tendency of this result is the same as that of the result obtained by the crack growth tests, as shown in Figs. 4 and 5. Based on these results, the values of $\Delta K_{\text {eff,th }}$ are considered to be nearly constant, irrespective of the type of experimental method, material, and stress ratio within the range of this study. Moreover, it is considred that the values of $\Delta K_{t h}$ differ under these conditions because the values of $K_{c l, t h}$ also differ under the same conditions. In general, the value of $\Delta K_{\text {eff,th }}$ is considered to be constant for structural steels as well as spheroidal cast irons ${ }^{(20)(21)}$. However, it is reported that the value of $\Delta K_{\text {eff, } \text {, }}$ decreases remarkably under the conditions of a small crack, high $H V$, and extremely high $R^{(20)}$. Although the materials used in this study do not have a high hardness, further studies on the effect of the stress ratio, which includes extremely high $R$ value, on $\Delta K_{t h}$ are necessary.

\subsection{Difference in crack growth and tension-compression fatigue tests with regard to} crack closure behavior

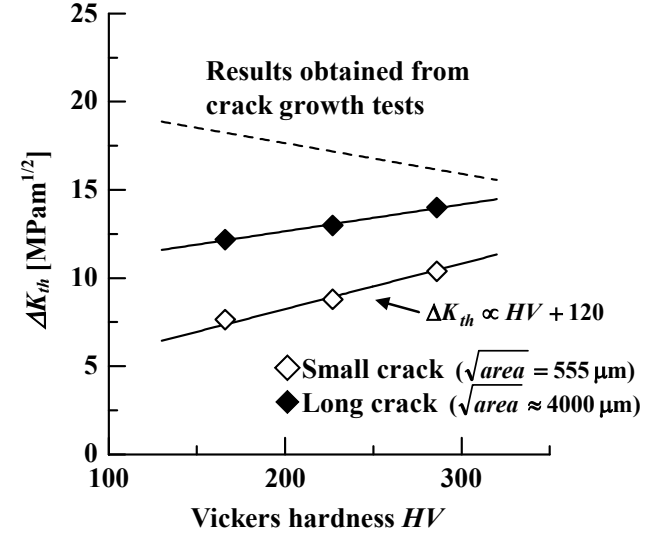

Fig.8 Relationship between $\Delta K_{\text {th }}$ and $H V$ for tension-compression fatigue tests at $R=-1$

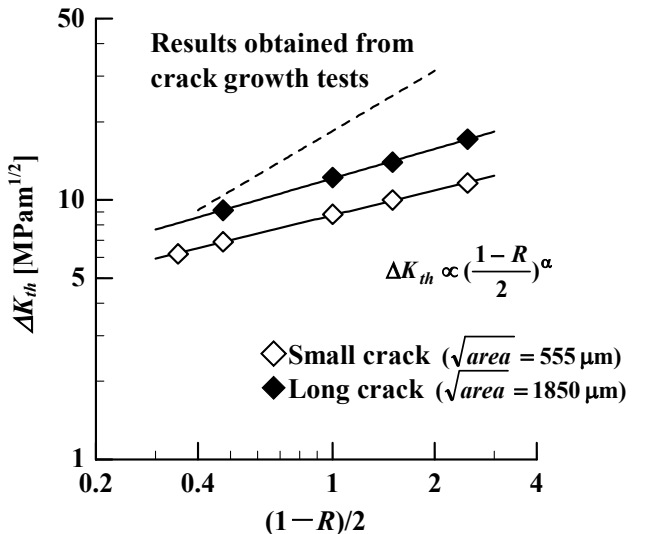

Fig.9 Relationship between $\Delta K_{t h}$ and $R$ for tension-compression fatigue tests at $R=-1$ 
As mentoned above, the difference in the crack growth and tension-compression fatigue tests with respect to $\Delta K_{t h, \infty}$ is due to the difference in the crack closure. To clarify the reason for the difference, the fracture surfaces of the specimens were observed.

Figure 11 shows the optical microscope images of the fracture sufaces of the specimens for the crack growth tests. These images show the sites around the center of the specimens, and the tips of the non-propagating cracks are denoted by dotted lines. All the fracture surfaces at the sites behind the tips of the non-propagating cracks are darkened. A comparison of the fracture surfaces of the FP, F, and $\mathrm{P}$ materials with $R=-1$ reveals that the lower the hardness, the darker is the fracture surface. The condition of these fracture surfaces corresponds to the values of $K_{c l}$. In other words, the higher the values of $K_{c l}$, the darker is the fracture surface. Moreover, the darkened fracture surfaces depend on $R$ and $H V$. A comparison between Figs. 11 (a) and (b) reveals that the fracture surfaces darken with a decrease in the stress ratio. In contrast, such darkened surfaces were not observed in the tension-compression fatigue tests, irrespective of the crack length and stress ratio. For the FP material, the values of $K_{c l, t h}$ obtained by the crack growth test, which was conducted by gradually decreasing $\Delta K$, were approximately 1.8 and 1.3 times higher than those obtained by the tension-compression fatigue tests, which was conducted by gradually increasing $\Delta K$. These results indicate that the darker the fracture surface, the larger is the difference in $K_{c l, t h}$.

Figure 12 shows the fracture surfaces of the FP material tested at $R=-1.5$ and $R=0.05$ and observed using an electron probe microanalyzer. Oxiden was observed in the area corresponding to the darkened fracture surfaces and oxide layers, which are considered to be produced by fretting, are deposited on these surfaces. From this observation, it is considered that the crack closure is developed due to the oxide layers produced by fretting in the crack growth tests; consequently, the values of $K_{c l}$ are larger than those in the $\Delta K$-increasing tests, i.e., the tension-compression fatigue tests. In addition, it has been reported that graphite crushed on the fracture surface ${ }^{(22)}$ of spheroidal cast irons induces crack closure. However, since such a surface containing crushed graphite cannot be observed on the fracture, as inferred from the carbon element distribution shown in Fig. 12, the effect of the graphiteinduced crack closure is considered to be small. Furthermore, it is reported that the surface roughness on a fracture surface promotes oxide-induced crack closure caused by fretting ${ }^{(23)}$. However, since the oxide layers are deposited on a large area of the fracture surface in the crack growth tests conducted in this study, the difference in the surface roughness cannot be evaluated quantitatively. Based on these results, the effects of $H V$ and $R$ on $\Delta K_{t h, \infty}$ of the specimens obtained from and tension-compression fatigue tests are investigated.

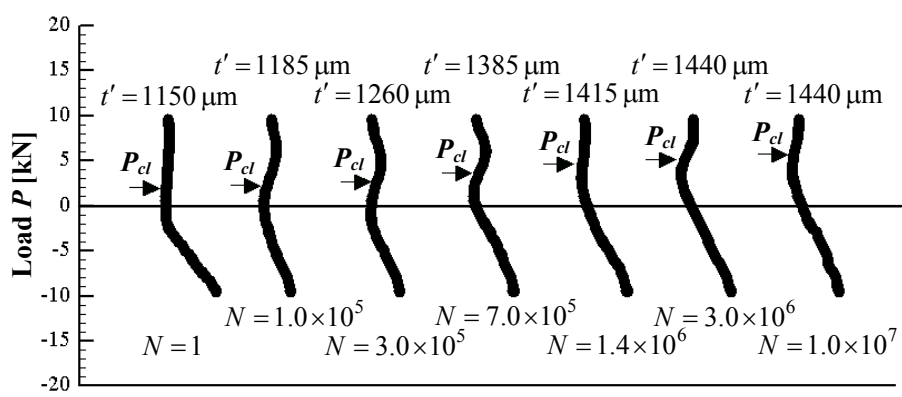

Fig.10 Behavior of crack closure at fatigue limit in the specimen having a long crack for FP material (Notch depth $t=1 \mathrm{~mm}$, Stress ratio $R=-1$ )

Table3 $\Delta K_{t h}, K_{c l, t h}$, and $\Delta K_{\text {eff,th }}$ obtained from tension-compression fatigue tests for FP material $[\mathrm{MPa} \sqrt{\mathrm{m}}]$

\begin{tabular}{c|c|c|c|c}
\hline Specimen & Stress ratio & $\Delta K_{\text {th }}$ & $K_{\text {cl,th }}$ & $\Delta K_{\text {eff } t \text { th }}$ \\
\hline Small crack & $R=-1$ & 10.01 & 2.15 & 2.86 \\
\cline { 2 - 5 }$(t=200 \mu \mathrm{m})$ & $R=-1.5$ & 10.93 & 1.46 & 2.92 \\
\hline $\begin{array}{c}\text { Long crack } \\
(t=1 \mathrm{~mm})\end{array}$ & $R=-1$ & 14.71 & 4.26 & 3.10 \\
\cline { 2 - 5 } & $R=-1.5$ & 16.16 & 3.42 & 3.04 \\
\hline
\end{tabular}


As mentioned above, the effects of $H V$ and $R$ on $\Delta K_{t h, \infty}$ for long cracks obtained in the $\Delta K$-increasing tests are similar to those on $\Delta K_{t h}$ for small cracks. In the case of a small crack, since the condition of small scale yielding is generally not satisfied, it is considered that crack closure is not developed and consequently the crack remains open for a wide range of loading conditions. Therefore, the values of $\Delta K_{t h}$ for such a small crack are considered to be influenced by the average resistance of a material against plastic deformation as the first approximation, since the fatigue limit of smooth specimens without a crack generally has a good correlation with $H V$. For a long crack, the effects of oxide-induced crack closure by fretting and plastic-induced crack closure are considered, which are thought to be very small for a small crack and tend to increase as the material gets softer. However, the effects are considered to be relatively small for a long crack obtained from $\Delta K$-increasing tests. Therefore, it is considered that the tendency of the effects of $H V$ and $R$ on $\Delta K_{t h, \infty}$ for a long crack obtained by the $\Delta K$-increasing tests is similar to that of the effects on $\Delta K_{t h}$ for a small crack.

In contrast, with regard to the crack growth tests, the values of $\Delta K_{t h, \infty}$ were measured by gradually decreasing $\Delta K$. Therefore, the crack closure was generated before reaching $\Delta K_{t h, \infty}$. Figure 13 shows the changes in $K_{c l}$ in the $\Delta K$-increasing and $\Delta K$-decreasing tests. The change in $K_{c l}$ in the $\Delta K$-decreasing test indicates that the crack closure $K_{c l}$ did not decrease, although $\Delta K$ gradually decreased. Instead, the value of $K_{c l}$ increased due to the formation of an oxide layer by fretting. The effect of the oxide layer on the crack closure is large, and the value of $K_{c l, t h}$ in the $\Delta K$-decreasing tests is sometimes 1.8 times higher than that in the $\Delta K$ increasing tests, as mentioned above. Thus, since the effect of the oxide-induced crack closure on $\Delta K_{t h}$ in the $\Delta K$-decreasing tests is larger than that in the $\Delta K$-increasing tests, it is believed that the tendency of the effects of $H V$ and $R$ on $\Delta K_{t h}$ in the $\Delta K$-decreasing tests is dfferent from that of the effects in the $\Delta K$-increasing tests.

In general, the tendency of $\Delta K_{t h, \infty}$ in the $\Delta K$-decreasing tests to decrease as the material becomes harder is attributed to the fact that fretting is generated more easily as the material becomes softer, although fretting, which could be the main cause of the development of crack closure, is not necessarily dependent on only $H V$. With regard to the effect of $R$ on $\Delta K_{t h, \infty}$, since fracture surfaces are often in contact with each other over a wide range of loading conditions, the lower the stress ratio, the easier it is to generate fretting. Therefore, it is considered that $\Delta K_{t h, \infty}$ at a low $R$ value increases remarkably and its tendency is different from that in the $\Delta K$-increasing tests.

Direction of crack growth

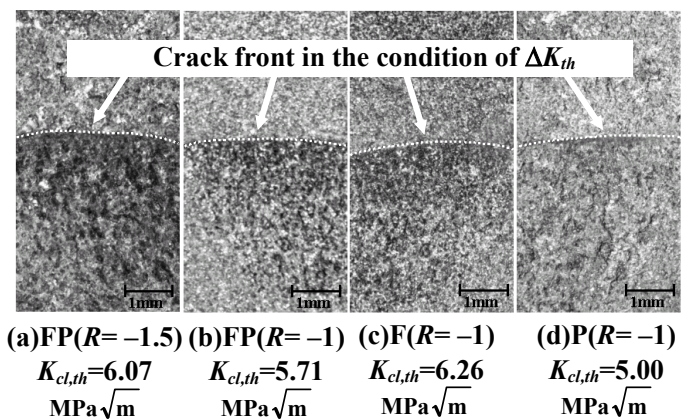

Fig.11 Photomicrographs of fracture surfaces on specimens for crack growth tests

(b) $\boldsymbol{R}=\mathbf{0 . 0 5}$
Fig.12 EPMA analysis around the crack front

at $\Delta K_{\text {th }}$ on FP material for crack growth tests 


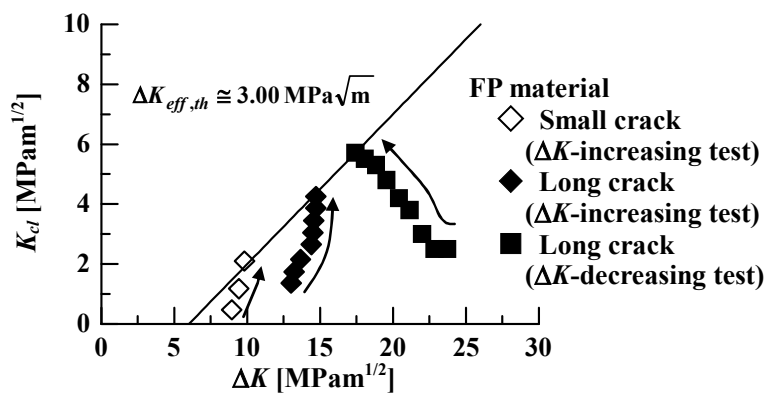

Fig.13 Crack closure behavior of FP material for $\Delta K$-increasing tests and $\Delta K$-decreasing tests at $R=-1$

Based on these results, the application of prediction equations using $\Delta K_{t h, \infty}$ and $\alpha$ obtained by the $\Delta K$-decreasing tests results in the overestimation of the fatigue strength of shot-blasted ductile cast irons with cast surfaces, which contain large defects and residual stress. Therefore, the fatigue limit evaluation of these materials should employ $\Delta K_{t h, \infty}$ and $\alpha$ obtained by the $\Delta K$-increasing tests, as shown in this study. For the range of stress ratios tested in this study, Eq. (7) can be used, to predict the fatigue limit of not only the shot-blasted ferrite-pearlitic ductile cast irons having a small crack but also of those having a long crack with sufficient accuracy for practical use.

\section{Conclusion}

In this study, the threshold stress intensity factors, $\Delta K_{t h} \mathrm{~s}$, for specimens with small and long cracks were estimated by tension-compression fatigue tests, which were conducted by gradually increasing $\Delta K$, and crack growth tests, which were conducted by gradually decreasing $\Delta K$. Further, the effects of hardness $(H V)$ and stress ratio $(R)$ on $\Delta K_{t h}$ were investigated from the viewpoint of crack closure behavior. The following results were obtained:

(1) The effects of $H V$ and $R$ on $\Delta K_{t h}$ for specimens with a small crack can be successfully evaluated by applying the $\sqrt{\text { area }}$ parameter model.

(2) With respect to the effect of $H V$ and $R$ on the threshold stress intensity factor range of specimens with a long crack, $\Delta K_{t h, \infty}$, the tendency of $\Delta K_{t h, \infty}$ in the $\Delta K$-decreasing tests is clearly different from that of $\Delta K_{t h, \infty}$ in the $\Delta K$-increasing tests.

(3) The values of $\Delta K_{t h, \infty}$ obtained by the $\Delta K$-increasing tests increase as the material becomes harder, and the effect of $R$ on $\Delta K_{t h, \infty}$ obtained by the $\Delta K$-increasing tests is similar to that on $\Delta K_{\text {th }}$ of specimens with a small crack.

(4) The difference in the $\Delta K$-decreasing and $\Delta K$-increasing tests with respect to $\Delta K_{t h, \infty}$ depends on the difference in their crack closure. Further, the crack closure of the specimens obtained under the $\Delta K$-decreasing tests develops remarkably due to an oxide layer on the fracture surface produced by fretting.

\section{Acknowledgements}

We thank Prof. Y. Murakami of the Kyushu University and N. Nakajima of ASAHI TEC Corporation for their thoughtful and helpful comments.

\section{References}

(1) Yamabe, J., et al., Evaluation of Fatigue Strength of Ductile Cast Iron Composed Ferrite and Pearlitic Structures with As-cast surface (Effect of Surface Roughness and Microstructure Transition Layers on Fatigue Strength), Trans. J. Soc. Mech. Eng. A, Vol. 71, No. 702 (2005), pp. 262-270.

(2) Yamabe, J., et al., Evaluation of Fatigue Strength of Ductile Cast Iron Composed Ferrite and Pearlitic Structures with casting surface (Effect of Surface Roughness, Microstructure 
Transition Layers and Defects on Fatigue Strength), Trans. J. Soc. Mech. Eng. A, Vol. 71, No. 701 (2005), pp. 116-123.

(3) Murakami, Y., Endo, M., A Geometrical Parameter for the Quantitative Estimation of the Effects of Small Defects on Fatigue Strength of Metals, Trans. J. Soc. Mech. Eng. A, Vol. 49, No. 438 (1983), pp. 127-136.

(4) Murakami, Y., Endo, M., Effect of Hardness and Crack Geometry on $\Delta K_{t h}$ of small cracks, J. Soc. Mater. Sci., Jpn., Vol. 35, No. 395 (1986), pp. 911-917.

(5) Murakami, Y., Metal Fatigue: Effects of Small Defects and Nonmetallic Inclusions (2002), Elsevier.

(6) Miyazaki, T., et al., Quantitative Evaluation of Fatigue Limit of a Metal with a Arbitrary Crack under a Stress Controlled Condition, Trans. J. Soc. Mech. Eng. A, Vol. 68, No. 673 (2002), pp. 1352-1359.

(7) Kondo, Y., et al., The effect of material hardness and mean stress on the fatigue limit of steels containing small defects, Fatigue Fract. Engng. Mater. Struct., Vol. 26 (2003), pp. 675-682.

(8) Kawakami, Y., et al., Study on Fatigue Strength of SCM440 Steel under High Mean Stress, Trans. J. Soc. Mech. Eng. A, Vol. 69, No. 678 (2003), pp. 396-403.

(9) Japan Welding Society, WES2805 (1997).

(10) Nakai, Y., et al., Stress Ratio Effect on Fatigue Crack Growth Threshold in Steels, $J$. Soc. Mater. Sci., Jpn., Vol. 33, No. 371 (1984), pp. 1045-1051.

(11) Klesnil, M. and Lukas, P., Effect of stress cycle asymmetry of fatigue crack growth, Mat. Sci. Eng., Vol. 9 (1972), pp. 231-240.

(12) Horikawa, T., et al, Y., Effect of Stress Ratio on Fatigue Strength of Micro Pulse Induction Hardness Steel with Very Thin Case-Hardened Depth, J. Soc. Mater. Sci., Jpn., Vol. 46, No. 11 (1997), pp. 1242-1247.

(13) Zhang, L., et al., Effect of Stress Ratio on Fatigue Threshold of Cracked Components, Trans. J. Soc. Mech. Eng. A, Vol. 64, No. 621 (1998), pp. 1221-1228.

(14) ASTM standard E 647-95a (1995).

(15) Kikukawa, M., et al., Study on Test Method of Fatigue Crack Growth Threshold, J. Soc. Mater. Sci., Jpn., Vol. 30, No. 330 (1981), pp. 276-282.

(16) Toyosada, M. and Niwa, T., Prediction of fatigue life for steel structures (2001), pp. 92, Kyouritu Syuppan Co. LTD.

(17) Murakami, Y., editor-in-chief, Stress Intensity Factors Handbook, Vol. 1 (1987), Pregamon Press.

(18) Ogawa, T., et al., Fatigue Crack Growth of Spheroidal-Graphite Cast Irons with Different Microstructures, Trans. J. Soc. Mech. Eng. A, Vol. 58, No. 554 (1992), pp. 1772-1778.

(19) Ogawa, T., et al., Fatigue Crack Propagation Characteristics of Spheroidal Graphite Cast Irons with Various Dual Phase Matrix Microstructures, Trans. J. Soc. Mech. Eng. A, Vol. 56, No. 523 (1990), pp. 482-487.

(20) Kondo, Y., et al., Large Reduction in the $\left(\Delta K_{\text {eff }}\right)_{t h}$ of Short Crack at Extremely High Stress Ratio, J. Soc. Mater. Sci., Jpn., Vol. 53, No. 6 (2004), pp. 661-666.

(21) Tanaka K., Mechanics Approach to Small Fatigue Crack Propagation, Trans. J. Soc. Mech. Eng. A, Vol. 54, No. 497 (1985), pp. 1-7.

(22) Ogawa, T., et al., Threshold Characteristics and Crack Closure of Fatigue Crack Growth of Spheroidal Graphite Cast Iron FCD450 (in Japanese), Trans. J. Soc. Mech. Eng. A, Vol. 51, No. 467 (1984), pp. 1660-1667.

(23) Sugiyama, Y., et al., Difference in Fatigue Crack Propagation Behavior at Stage II between Spheroidal Graphite Cast Iron and High Strength Steel, J. Soc. Mater. Sci., Jpn., Vol. 37, No. 418 (1987), pp.776-782. 